\title{
O PAPEL DA TOMOGRAFIA COMPUTADORIZADA NO ESTADIAMENTO E TRATAMENTO CIRÚRGICO DO CARCINOMA EPIDERMÓIDE DO SEIO PIRIFORME
}

\author{
THE ROLE OF COMPUTED TOMOGRAPHY ON SURGICAL TREATMENT OF \\ SQUAMOUS CELL CARCINOMA OF THE PYRIFORM SINUS
}

\author{
Richard Volpato ${ }^{1}$; Ricardo Pires de Souza²; Abrão Rapoport, ECBC - SP; \\ Paulo Bentes de Carvalho Neto ${ }^{4}$; Ivo Marquis Beserra Júnior ${ }^{4}$
}

\begin{abstract}
RESUMO: Objetivo: Determinar a real extensão das neoplasias do seio piriforme através da tomografia computadorizada após estadiamento clínico/endoscópico (seio piriforme, prega ariepiglótica, espaço paraglótico, glote, subglote, orofaringe, cartilagem tireóide, cartilagem cricóide, cartilagem aritenóide, esôfago cervical, extensão para tecidos moles extralaríngeos e musculatura prévertebral) e sua repercussão no planejamento cirúrgico. Método: O estudo incluiu pacientes portadores de carcinoma epidermóide de seio piriforme, atendidos no Departamento de Cabeça e Pescoço e Otorrinolaringologia do Hospital Heliópolis, Hosphel, São Paulo de 1988 e 2003. Foram avaliados os prontuários de 31 pacientes, sendo 29 (93,5\%) do sexo masculino e dois $(6,5 \%)$ do sexo feminino. A análise das tomografias foi realizada por três radiologistas individualmente e para o estudo da concordância interobservadores, foi utilizado o índice Kappa. Resultados: A TC apresentou: forte concordância na avaliação de seio piriforme, prega ariepiglótica, espaço paraglótico e subglote; boa concordância para a orofaringe, glote, cartilagem cricóide, esôfago cervical e tecidos moles extralaríngeos; moderada para as cartilagens tireóide e aritenóide; fraca no estudo da musculatura pré-vertebral. Conclusão: A avaliação interobservadores das imagens do CEC do seio priforme determina o re-estadiamento TNM e conseqüente mudanças do paradigma cirúrgico (Rev. Col. Bras. Cir. 2007; 34(2): 78-83).
\end{abstract}

Descritores: Estadiamento de neoplasias; Tomografia; Carcinoma de células escamosas; Procedimentos cirúrgicos operatórios.

\section{INTRODUÇÃO}

As neoplasias malignas da hipofaringe representam 5 a $10 \%$ dos tumores das vias aero-digestivas superiores, sendo que $90 \%$ são carcinomas epidermóides e o seu prognóstico depende de uma correta avaliação da disseminação loco-regional ${ }^{1-5}$. Visto que o comprometimento mucoso superficial do câncer da hipofaringe é bem avaliado pelas laringoscopias direta e indireta, enquanto que a extensão profunda é melhor avaliada através de imagens seccionais. Estas imagens são obtidas através da tomografia computadorizada (TC) e da ressonância nuclear magnética (RM). Estes exames complementares à endoscopia, são indispensáveis para um correto planejamento terapêutico ${ }^{6-9}$, possibilitando um reestadiamento prévio que atenda às necessidades resgate terapêutica do paciente com carcinoma espinocelular (CEC) do seio piriforme.

Assim sendo, a partir do estadiamento clínico conjunto da TNM das neoplasias do seio piriforme e das imagens obtidas através da TC, é possível estabelecer um planejamento terapêutico adequado, onde a imagem depende da sensibilidade do método, de sua especificidade, eficácia, reprodutibilidade e consistência da interpretação dos obser- vadores ${ }^{10-12}$. Isto permite objetivar que o planejamento cirúrgico isolado ou associado à radioterapia atenda às expectativas de sobrevida e cura dos pacientes com carcinoma epidermóide de hipofaringe.

\section{MÉTODO}

O estudo incluiu 31 pacientes portadores de CEC do seio piriforme atendidos de 1998 a 2003 nos Departamentos de Cirurgia de Cabeça e Pescoço/ORL e Diagnóstico por Imagem do Hospital Heliópolis, HOSPHEL, São Paulo, Brasil, sendo o projeto de pesquisa aprovado no Comitê na Pesquisa $\operatorname{sob~n}^{\circ} 270(13 / 2 / 2004)$.

Como critérios de inclusão no estudo tivemos: estadiamento pelo TNM (1997) através da laringoscopia direta ou indireta estádio IV; diagnóstico histopatológico de CEC; tomografia computadorizada prévia ao início do tratamento.

Para a realização da TC foi utilizado o aparelho Toshiba- TCT-500S. O paciente era colocado em posição supina, com o pescoço em extensão, e solicitado a não deglutir e a manter uma respiração tranqüila. Inicialmente foram realizados cortes axiais de espessura e incremento de $5 \mathrm{~mm}$,

1. Mestre pelo Curso de Pós-Graduação em Ciências da Saúde do Hospital Heliópolis, HOSPHEL - São Paulo.

2. Radiologista do Departamento de Diagnóstico por Imagem do Hospital Heliópolis, HOSPHEL - São Paulo.

3. Cirurgião do Departamento de Cirurgia de Cabeça e Pescoço e Otorrinolaringologia do Hospital Heliópolis, HOSPHEL - São Paulo.

4. Mestrando pelo Curso de Pós-Graduação em Ciências da Saúde do Hospital Heliópolis, HOSPHEL - São Paulo.

Recebido em 04/09/2006

Aceito para publicação em 06/11/2006

Conflito de interesses : nenhum

Fonte de financiamento: nenhuma

Trabalho realizado no Hospital Heliópolis, HOSPHEL - São Paulo. 
estendendo-se do ângulo da mandíbula até a transição cérvico-torácica, orientados por meio de radiografia digital em perfil. Todas as aquisições eram realizadas após a injeção endovenosa de material contraste iodado, na dose de 1 a 2, $0 \mathrm{ml} /$ $\mathrm{kg}$, com concentração de 60 a 76\%. Para análise das imagens, três profissionais titulares do Colégio Brasileiro de Radiologia, sem o conhecimento prévio do diagnóstico clínico, endoscópico e histopatológico, participaram do processo de análise interobservadores.

Foram avaliadas imagens de extensões tumorais do seio piriforme para a orofaringe, espaços paraglóticos, pregas, ariepiglóticas, glote, subglote, cartilagens (tireóide, cricóide, aritenóide), esôfago cervical, musculatura pré-vertebral e tecidos moles extralaríngeos. Para avaliar o comprometimento das estruturas profundas, foram aferidos: o borramento do tecido gorduroso, efeito de massa local e envolvimento direto da lesão quando havia presença da neoplasia com densidade das partes moles na região estudada, verificando a invasão das cartilagens, quando havia lesão interna e externamente à estrutura presença de erosão ou esclerose, músculos pré-vertebrais comprometidos quando havia obliteração do tecido gorduroso entre a massa e a fáscia pré-vertebral ou envolvimento direto da musculatura com espessamento assimétrico.

Tabela 1 - Interpretação do coeficiente Kappa.

\begin{tabular}{ll}
\hline Coeficiente Kappa $(\mathbf{K})$ & Concordância \\
\hline $\mathrm{K}<0,20$ & Não há \\
$0,20 \leq \mathrm{K}<0,40$ & Fraca \\
$0,40 \leq \mathrm{K}<0,60$ & Moderada \\
$0,60 \leq \mathrm{K}<0,80$ & Boa \\
$0,80 \leq \mathrm{K} \leq 1,00$ & Forte \\
\hline
\end{tabular}

Fonte: Everitt, $1982^{13}$
Para estruturas medianas ímpares (orofaringe, glote, subglote, cartilagem tireóide, cartilagem cricóide, esôfago cervical, musculatura pré-vertebral e tecidos moles extra faríngeos), o "n” foi igual ao número de casos estudados. Nos casos de estruturas pares bilaterais (SP, espaço paraglótico, prega ariepiglótica e cartilagem aritenóide), o "n” foi duplicado (lado direito e esquerdo).

Quanto ao método estatístico, para a avaliação da concordância interobservadores foi utilizado o índice de Kappa $(\mathrm{K})^{13}$, com os critérios de concordância mostrados na tabela 1 . O software utilizado foi o SPSS, versão 10.0.

\section{RESULTADOS}

Foram encontrados: $18 \mathrm{~T}_{4}, 12 \mathrm{~T}_{3}, 1 \mathrm{~T}_{2}, 5 \mathrm{~N}_{0}, 9 \mathrm{~N}_{1}$, $9 \mathrm{~N}_{2} 8 \mathrm{~N}_{3}$ sendo 17 estádio III e 14 estádio IV.

O resultado da análise das imagens entre os observadores está expresso nas tabelas abaixo: seio piriforme, prega ariepiglótica e espaço paraglótico (tabela 2); orofaringe, glote e subglote (tabela 3); cartilagens: tireóide, cricóide e aritenóide (tabela 4); esôfago cervical, músculos pré-vertebrais e tecidos moles extrafaríngeos (tabela 5).

\section{DISCUSSÃO}

\section{Seio piriforme}

As neoplasias malignas do seio piriforme têm evolução insidiosa, sendo raro o seu diagnóstico clínico nos estádios iniciais, fato este aferido em nossa casuística onde diagnosticamos 17 estadios III e 14 estádios IV concordante com a literatura ${ }^{14-17}$. Embora a propedêutica clínica evidencie a extensão mucosa da lesão, com a tomografia computadorizada é possível avaliar a extensão submucosa, tornando-se fundamental para o correto delineamento do planejamento terapêutico. Assim, torna-se

Tabela 2 - Análise do envolvimento de seio piriforme, prega ariepiglótica e espaço paraglótico segundo os observadores.

\section{Seio piriforme}

\begin{tabular}{llll} 
Observador & Kappa & Erropadrão & Kappamédio \\
\hline $1 \times 2$ & 1.000 (forte concordância) & 0.000 & \\
$1 \times 3$ & 0.930 (forte concordância) & 0.049 & 0.930 (forte concordância) \\
$2 \times 3$ & 0.930 (forte concordância) & 0.049 & \\
\hline
\end{tabular}

\section{Prega ariepiglótica}

\begin{tabular}{llll} 
Observador & Kappa & Erropadrão & Kappamédio \\
\hline $1 \times 2$ & 0.863 (forte concordância) & 0.066 & \\
$1 \times 3$ & 0.793 (boa concordância) & 0.080 & Kappa médio $=0.838$ (forte concordância) \\
$2 \times 3$ & 0.859 (forte concordância) & 0.068 &
\end{tabular}

\section{Espaço paraglótico}

\begin{tabular}{llll} 
Observador & Kappa & Erropadrão & Kappamédio \\
\hline $1 \times 2$ & 0.899 (forte concordância) & $1 \times 2$ & \\
$1 \times 3$ & 0.899 (forte concordância) & $1 \times 3$ & Kappa médio=0.921 (forte concordância) \\
$2 \times 3$ & 0.932 (forte concordância) & $2 \times 3$ & \\
\hline
\end{tabular}


Tabela 3 - Análise do envolvimento da orofaringe, glote e subglote.

\begin{tabular}{|c|c|c|c|}
\hline \multicolumn{4}{|l|}{ Orofaringe } \\
\hline Observador & Kappa & Erropadrão & Kappamédio \\
\hline $1 \times 2$ & 0.742(boa concordância) & 0.742 & \multirow{3}{*}{ Kappa médio $=0.742$ (boa concordância) } \\
\hline $1 \times 3$ & 0.807(forte concordância) & 0.106 & \\
\hline $2 \times 3$ & 0.678(boa concordância) & 0.132 & \\
\hline \multicolumn{4}{|l|}{$\overline{\text { Glote }}$} \\
\hline Observador & Kappa & Erropadrão & Kappamédio \\
\hline $1 \times 2$ & 0.685 (boa concordância) & 0.139 & \multirow{3}{*}{ Kappa médio $=0.672$ (boa concordância) } \\
\hline $1 \times 3$ & 0.648 (boa concordância) & 0.143 & \\
\hline $2 \times 3$ & 0.675(boa concordância) & 0.139 & \\
\hline \multicolumn{4}{|l|}{$\overline{\text { Subglote }}$} \\
\hline Observador & Kappa & Erropadrão & Kappamédio \\
\hline $1 \times 2$ & 0.839 (forte concordância) & 0.156 & \multirow{3}{*}{ Kappa médio $=0.841$ (forte concordância) } \\
\hline $1 \times 3$ & 0.716 (boa concordância) & 0.186 & \\
\hline $2 \times 3$ & 0.870 (forte concordância) & 0.127 & \\
\hline
\end{tabular}

mandatória a avaliação radiológica da extremidade proximal do esôfago, espaço paraglótico, tecidos extralaríngeos e invasão da musculatura pré-vertebra ${ }^{17}$. Isto através de análise inter-observadores permite avaliar o grau concordância entre os avaliadores através do índice de Kappa médio. Em nossa casuística, tivemos um Kappa médio fortemente concordante de 0,953 (Tabela 2) o que permite afirmar que nos estádios III e IV a análise interobservadores é relevante para a segurança da proposta terapêutica, o que permite uma indicação adequada da faringolaringectomia via de regra associada ao esvaziamento radical uni ou bilateral.

\section{Prega ariepiglótica}

Considerando que esta estrutura limita medialmente o seio piriforme, e lembrando que as neoplasias desta estrutura tem habitualmente um grande componente reacional (edema) a tomografia computadorizada contribui com imagens que, permite uma análise fortemente concordante com Kappa médio de 0,838 (Tabela 2) limitando a incidência de avaliações falso-positivas decorrentes da superavaliações conseqüentes ao edema discreto ${ }^{18}$. Todavia, a presença do componente inflamatório induz o cirurgião ao aumento da margem de segurança na mucosa faríngea, fato este que pode ser alterado pela concordância interobservadores da real extensão da neoplasia.

Tabela 4 - Análise do envolvimento das cartilagens tireóide, cricóide, aritenóide.

\begin{tabular}{llll}
\hline $\begin{array}{l}\text { Tireóide } \\
\text { Observador }\end{array}$ & Kappa & Erropadrão & Kappamédio \\
\hline $1 \times 2$ & 0.638 (boa concordância) & 0.137 & \\
$1 \times 3$ & 0.426 (moderada concordância) & 0.164 & Kappa médio $=0.493$ (moderada concordân- \\
cia) & & & \\
$2 \times 3$ & 0.418 (moderada concordância) & 0.184 & \\
\hline
\end{tabular}

\section{Cricóide}

\begin{tabular}{llll} 
Observador & Kappa & Erropadrão & Kappamédio \\
\hline $1 \times 2$ & 0.571 (moderada concordância) & 0.158 & \\
$1 \times 3$ & 0.636 (boa concordância) & 0.158 & Kappa médio $=0.710$ (boa concordância) \\
$2 \times 3$ & 0.923 (forte concordância) & 0.075 & \\
\hline
\end{tabular}

\section{Aritenóide}

\begin{tabular}{llll} 
Observador & Kappa & Erropadrão & Kappamédio \\
\hline $1 \times 2$ & 0.611 (boa concordância) & 0.114 & \\
$1 \times 3$ & 0.438 (moderada concordância) & 0.098 & Kappa médio $=0.551$ (moderada concordância) \\
$2 \times 3$ & 0.606 (boa concordância) & 0.098 & \\
\hline
\end{tabular}


Tabela 5 - Análise do envolvimento do esôfago cervical, músculos pré-vertebrais, tecidos moles extrafaríngeos.

\begin{tabular}{llll}
\hline $\begin{array}{l}\text { Esôfago cervical } \\
\text { Observador }\end{array}$ & Kappa & Erropadrão & Kappamédio \\
\hline $1 \times 2$ & 0.595 (moderada concordância) & 0.159 & \\
$1 \times 3$ & 0.661 (boa concordância) & 0.154 & Kappa médio = 0.675 (boa concordância) \\
$2 \times 3$ & 0.769 (boa concordância) & 0.126 & \\
\hline Músculos pré-vertebrais & & \\
Observador & Kappa & Erropadrão & Kappamédio \\
\hline $1 \times 2$ & 0.518 (moderada concordância) & $1 \times 2$ & \\
$1 \times 3$ & 0.168 (não há concordância) & $1 \times 3$ & Kappa médio $=0.367$ (fraca concordância) \\
$2 \times 3$ & 0.415 (moderada concordância) & $2 \times 3$ & \\
\hline
\end{tabular}

Tecidos moles extrafaríngeos

\begin{tabular}{llll} 
Observador & Kappa & Erropadrão & Kappamédio \\
\hline $1 \times 2$ & 0.729 (boa concordância) & 0.125 & \\
$1 \times 3$ & 0.665 (boa concordância) & 0.136 & Kappa médio $=0.747$ (boa concordância) \\
$2 \times 3$ & 0.793 (boa concordância) & 0.113 & \\
\hline
\end{tabular}

\section{Espaço paraglótico}

Esta estrutura não é passível de avaliação através da laringoscopia, o que justifica a indicação da tomografia computadorizada $^{19}$, fato este que justificou em nosso estudo (Tabela 2) um índice de Kappa médio, fortemente positivo de 0,838. Para esta localização, a impossibilidade de avaliação endoscópica, reforça a indicação da tomografia computadorizada para melhor nortear o planejamento cirúgico.

\section{Orofaringe}

As neoplasias malignas do seio piriforme se extendem através da prega faringoepiglótica para a parede posterior da orofaringe, compromentendo habitualmente a valécula e a base da língua, justificando a indicação da tomografia computadorizada $^{18}$. Assim, na nossa avaliação houve boa concordância entre os observadores com Kappa médio (Tabela 3) de 0,742 . A invasão neoplásica da orofaringe é melhor avaliada com o auxílio das margens, considerado a necessidade da ressecção da base da língua ou loja amigdalina.

\section{Glote}

O acomentimento do ângulo anterior do seio piriforme explica a invasão da região glótica da laringe com comprometimento das cordas vocais e comissuras anterior e posterior ${ }^{20}$, havendo boa concordância (Tabela 3) com índice de Kappa médio de 0,672 entre os observadores.

\section{Subglote}

A invasão de subglote pelas neoplasias da hipofaringe ${ }^{18,20}$, caracterizada por um índice de Kappa médio (Tabela 3) de 0,841 fortemente concordante entre os observadores, permite afirmar que o estadiamento TNM destas neoplasias habitualmente se altera quando da avaliação das imagens tomográficas.
7, 8 e 9 Cartilagens tireóide, cricóide e aritenóide

O esqueleto cartilaginoso é constituído de cartilagem hialinas e o seu comprometimento aumenta o estadiamento das neoplasias do seio piriforme para $\mathrm{T} 4{ }^{19}$ ${ }^{21}$, influenciando o seu prognóstico e o planejamento terapêutico. Apesar da tomografia computadorizada não ter uma acurácia perfeita na avaliação do arcabouço cartilaginoso da tireóide, o seu emprego permitiu em nossa casuística a obtenção de um índice de Kappa médio de moderada concordância (Tabela 4) de 0,493 para a cartilagem tireóide, de boa concordância (Tabela 4) para a cartilagem cricóide de 0,710 e moderada concordância (Tabela 4) para a cartilagem aritenóide de 0,551. Isto permite concluir que as limitações clínicas na avaliação das neoplasias e seu estadiamento são corroboradas por imagens de pouca eficiência para a avaliação destas lesões.

\section{Esôfago cervical}

O estudo das neoplasias malignas da hipofaringe está associado à infiltração esofagiana, repercutindo no planejamento terapêutico ${ }^{22}$. Daí, o emprego da tomografia computadorizada em nossa casuística aferiu (Tabela 5) um Kappa médio de 0,675 de boa concordância entre os observadores, mas que pouco contribui para a mudança do estadiamento e da adequação terapêutica.

\section{Musculatura pré vertebral}

Em decorrência da freqüente invasão da musculatura pré-vertebral pelas neoplasias malignas da hipofaringe, o que contraindica o planejamento cirúrgico ${ }^{17,23}$, é relevante a indicação da tomografia computadorizada. Em nossa experiência, a avaliação interobservadores permitiu (Tabela 5) a obtenção de um Kappa médio de 0,367 de fraca concordância entre os avaliadores. 


\section{Tecidos moles extrafaríngeos}

A agressividade das neoplasias da hipofaringe explica sua usual disseminação para as estruturas extrafaríngeas ${ }^{22,23}$, o que torna a indicação da maior relevância. Em nosso grupo de estudo, obtivemos (Tabela 5) um kappa médio de 0,729 de boa concordância interobservadores).

Em síntese, podemos concluir que a indicação da tomografia computadorizada como complementação diagnóstica para o correto estadiamento TNM das neoplasias malignas da hipofaringe, foi fortemente concordante para o seio piriforme, prega ariepiglótica, espaço paraglótico e subglote. Para estas localizações, há necessidade do emprego de imagens obtidas da tomografia computadorizada para possibilitar um planejamento cirúrgico adequado quanto à sua extensão permitindo o estabelecimento real de critérios de cura e sobrevida (global e livre de doença $)^{24-27}$. Para as demais localizações, a análise interobservadores foi de relevância parcial no planejamento terapêutico destas neoplasias malignas.

\begin{abstract}
Background: The objective of this study is to determine the extension of pyriform sinus (PS), through CT after the clinical and endoscopic assessment. Methods: For this purpose, the following structures were ascertained: PS, aryepiglottic folds, paraglottic space, glottis, subglottis, pharynx, thyroid cartilage, cricoid cartilage, arytenoid cartilage, cervical esophagus, extralaringeal tumoral extension and prevertebral muscle invasion. We retrospectively studied 31 patients (29 men) evaluated in the Head and Neck Surgery Service of the Heliópolis Hospital, between 1988 and 2003. Results: Three radiologists were blinded to any clinical information, performed independent CT analysis determining the presence of involvement of each structure. The interobserver reproducibility was assessed by the Kappa rank. Strong interobserver reproducibility were found for pyriform sinus, aryepiglottic folds, paraglottic space and subglottis; good reproducibility for oropharynx, glottis, cricoid cartilage, cervical esophagus and extralaringeal tumoral extension; moderate reproducibility for thyroid and arytenoid cartilages; and there was only a weak reproducibility for prevertebral muscle invasion. Conclusion: The interobserver CT analyses of PSCC determine the TNM re-staging and changes in surgical paradigm.
\end{abstract}

Key words: Neoplasm staging ; Tomography; Carcinoma, Squamous Cell; Surgical Procedures, Operative.

\section{REFERÊNCIAS}

1. Gatenby RA, Mulhern CB, Strawitz J, Moldofsky PJ. Comparison of clinical and computed tomographic staging of head and neck tumors. AJNR Am J Neuroradiol. 1985;6(3):399401.

2. Righi PD, Kelley DJ, Ernst R, Deutsch MD, Gaskill-Shipley M, Wilson KM, Gluckman JL. Evaluation of prevertebral muscle invasion by squamous cell carcinoma. Can computed tomography replace open neck exploration? Arch Otolaryngol Head Neck Surg. 1996;122(6):660-3.

3. Pameijer FA, Mancuso AA, Mendenhall WM, Parsons JT, Kubilis PS. Can pretreatment computed tomography predict local control in T3 squamous cell carcinoma of the glottic larynx treated with definitive radiotherapy? Int J Radiat Oncol Biol Phys. 1997;37(5):1011-21.

4. Hirano M, Kurita S, Cho JS, Tanaka H. Computed tomography in determining laryngeal involvement of hypopharyngeal carcinoma. Ann Otol Rhinol Laryngol. 1998;97(5 Pt 1):476-82.

5. Hinerman RW, Amdur RJ, Mendenhall WM, Villaret DB, Robbins KT. Hypopharyngeal carcinoma. Curr Treat Options Oncol. 2002;3(1):41-9.

6. Aspestrand F, Kolbenstvedt A, Boysen M. Carcinoma of the hypopharynx: CT staging. J Comput Assist Tomogr. 1990;14(1):72-6.

7. Becker M. Oral cavity, oropharynx, and hypopharynx. Semin Roentgenol. 2000;35(1):21-30.

8. Souza RS. Diagnóstico por imagem dos tumores da laringe e da hipofaringe. In: Carvalho MB, editor. Tratado de cirurgia de cabeça e pescoço e otorrinolaringologia. $1^{a}$ ed. São Paulo: Atheneu; 2001. p.887-97.
9. Hermans R, Van der Goten A, Baert AL. Image interpretation in CT of laryngeal carcinoma: a study on intra- and interobserver reproducibility. Eur Radiol. 1997;7(7):1086-90.

10. Paiva RGS, Souza RP, Rapoport A, Soares AH. Avaliação por tomografia computadorizada do envolvimento loco-regional do carcinoma espinocelular de corda vocal. Radiol Bras. 2001;34(4):193-200.

11. Gusmão PDF, Mothes FC, Rubin LA, Gonçalves RZ, Telöken MA, schwartsmann CR. Avaliação da reprodutibilidade da classificação de Garden para fraturas do colo femoral. Rev Bras Ortop. 2002;37(9):381-6.

12. Obuchowski NA. How many observers are needed in clinical studies of medical imaging? AJR Am J Roentgenol. 2004;182(4):867-9.

13. Everitt BS. The analysis of contigency tables. $2^{\text {a }}$ ed. London: Chapman \& Hall; 1992.

14. Mancuso AA, Hanafee WN, Juillard GJ, Winter J, Calcaterra TC. The role of computed tomography in the management of cancer of the larynx. Radiology. 1977;124(1):243-4.

15. Mukherji SK, Pillsbury HR, Castillo M. Imaging squamous cell carcinomas of the upper aerodigestive tract: what clinicians need to know. Radiology. 1997;205(3):629-46.

16. Charlin B, Brazeau-Lamontagne L, Guerrier B, Leduc C. Assessment of laryngeal cancer: CT scan versus endoscopy. J Otolaryngol. 1988;18(6):283-8.

17. Sagel SS, AufderHeide JF, Aronberg DJ, Stanley RJ, Archer CR. High resolution computed tomography in the staging of carcinoma of the larynx. Laryngoscope. 1981;91(2):292-300.

18. Hermans R, Van der Bogaert W, Rijnders A, Baert AL. Value of computed tomography as outcome predictor of supraglottic squamous cell carcinoma treated by definitive radiation therapy. Int J Radiat Oncol Biol Phys. 1999; 44(4):755-65. 
19. Castelijns JA, Becker M, Hermans R. Impact of cartilage invasion on treatment and prognosis of laryngeal cancer. Eur Radiol. 1996;6(2):156-69.

20. Saleh EM, Mancuso AA, Stringer SP. Relative roles of computed tomography and endoscopy for determining the inferior extent of pyriform sinus carcinoma: correlative histopathologic study. Head Neck. 1993;15(1):44-52.

21. Keberle M, Kenn W, Hahn D. Current concepts in imaging of laryngeal and hypopharyngeal cancer. Eur Radiol. 2002;12(7):1672-83. Epub 2002 Feb 9.

22. Kazkayasi M, Önder T, Özkaptan Y, Can C, Pabuscu Y. Comparison of preoperative computed tomographic findings with postoperative histopathological findings in laryngeal cancers. Eur Arch Otorhinolaryngol. 1995;252(6):325-31.

23. Williams DW $3^{\text {rd }}$. Imaging of laryngeal cancer. Otolaryngol Clin North Am. 1997;30(1):35-58.

24. Kundel HL, Polansky M. Measurement of observer agreement. Radiology. 2003;228(2):303-8. Epub 2003 Jun 20.

25. Oktay MF, Cüreoglu S, Bukte Y, Yilmaz F, Tekin M, Osma U, Topçu I. The diagnosis accuracy of the clinical examination and the computed tomography findings in patients with laryngeal carcinoma who undergone total laryngectomy. Turch Arch Otolaryngol. 2002;40(3):189-95.

26. Catic D, Lourincevic A. Correlation of computed tomography and conventional hypopharyngography findings in hypopharyngeal tumours. Eur J Radiol. 1985 Aug;5(3):178-80.

27. King AD, Tse GM, Ahuja AT, Yuen EH, Vlantis AC, To EW, van Hasselt AC. Necrosis in metastatic neck nodes: diagnostic accuracy of CT, MR imaging, and US. Radiology. 2004;230(3):720-6.

Como citar este artigo:

Volpato R, Souza RP, Rapoport A, Carvalho Neto PB, Beserra Jr IM. O papel da tomografia computadorizada no estadiamento e tratamento cirúrgico do carcinoma epidermóide do seio piriforme. Rev Col Bras Cir. [periódico na Internet] 2007; 34(2). Disponível em URL: http://www.scielo.br/rcbc

Endereço para correspondência:

Abrão Rapoport - Rua Iramaia, $n^{\circ} 136$ - Jd. Europa

01450-020 - São Paulo-SP

E-mail: ararapoport@terra.com.br 